\title{
Working Memory Over a Six-Year Period in Young Binge Drinkers
}

Carbia, C. a , Cadaveira, F. ${ }^{\text {a }}$ López-Caneda, E. b, Caamaño-Isorna, F. c , Rodríguez Holguín, S. a, Corral, M. ${ }^{\mathrm{a}}$

${ }^{a}$ Department of Clinical Psychology and Psychobiology Universidade de Santiago de Compostela, Spain

${ }^{b}$ Neuropsychophysiology Lab, Research Center on Psychology (CIPsi), School of Psychology, University of Minho, Braga, Portugal

${ }^{\mathrm{c}}$ Consortium for Biomedical Research in Epidemiology \& Public Health (CIBERESP).

Department of Preventive Medicine, Universidade of Santiago de Compostela. Spain

Correspondence concerning this article should be addressed to Carina Carbia

Sinde, Department of Clinical Psychology and Psychobiology Universidade de Santiago de Compostela, Campus Universitario Sur, 15782, Santiago de Compostela, Spain. Tel: +34-8818-13915. Email: carina.carbia@usc.es 


\section{Abstract (256)}

Adolescence and early adulthood are periods of particular vulnerability to the neurotoxic effects of alcohol. Young people with alcohol use disorders display deficits in working memory (WM). This function is supported by the prefrontal cortex, a late-maturing brain region. However, little is known about the progression of cognitive dysfunctions associated with a binge drinking (BD) pattern of alcohol consumption among community adolescents. The objective of this study was to analyze the relationship between BD trajectory and WM in university students. An initial sample of 155 male and female first-year university students was followed prospectively over six years. The participants were classified as stable nonBDs, stable BDs and Ex-BDs according to the third item of the Alcohol Use Disorders Identification Test (AUDIT). WM was assessed using the Self-Ordered Pointing Task. Generalized linear mixed models were applied. The results showed that stable BDs committed more total perseverative errors and showed a lower WM span in the difficult blocks than stable non-BDs. Difficulties in WM span showed some improvement, whereas perseveration errors remained constant throughout the follow-ups in the stable BDs. There were no significant differences between Ex-BDs and non-BDs. In conclusion, stable BD is associated with WM deficits, particularly perseverations and low WM span in demanding trials, when compensatory mechanisms may no longer be successful. The partial improvement in WM span may support the notion of a neuromaturational delay, whereas the temporal stability of perseveration deficits may reflect either neurotoxic effects of alcohol or premorbid characteristics. Abandoning the BD pattern of alcohol consumption may lead to partial recovery.

Key words: adolescence; alcohol; working memory; longitudinal; heavy drinking 


\section{Introduction}

Alcohol is by far the most commonly used drug worldwide and is the third most important risk factor for disease (Rehm et al., 2009). Binge drinking (BD) is an increasingly prevalent pattern of alcohol consumption among European adolescents. This pattern is defined as the consumption of 4 or more drinks for women and 5 or more drinks for men in about 2 hours, leading to a blood alcohol concentration (BAC) of $0.8 \mathrm{~g} / 1$ (National Institute on Alcohol Abuse and Alcoholism [NIAAA], 2004). Recent reports indicate that BD is a common pattern of alcohol consumption in a third of European and American young people (Eurobarometer, 2010; Substance Abuse and Mental Health Services Administration [SAMHSA], 2013). BD is associated with an array of negative consequences and constitutes a major concern in many countries (Marshall, 2014).

Adolescence is a critical developmental period, which may contribute to a heightened vulnerability to the harmful effects of BD at this time (Bava \& Tapert, 2010). Neuromaturational changes during this period (Rubia, 2013) lead to significant improvements in complex cognitive functions, the so-called "executive functions" such as working memory (WM) (Diamond, 2013). These higher-level cognitive functions are mainly supported by functional networks involving fronto-striatal pathways and the prefrontal cortex (PFC) (Fuster, 2001). The relative immaturity of these areas may make them particular targets for the deleterious effects of ethanol (Petit, Maurage, Kornreich, Verbanck, \& Campanella, 2014).

Models of BD in adolescent animals induce frontal cortical degeneration (Crews et al., 2000), neuroinflammation (Pascual, Pla, Miñarro \& Guerri, 2014) and reductions in myelin in the PFC (Vargas, Bengston, Gilpin, Whitcomb, \& Richardson, 2014). In humans, young people with Alcohol Use Disorder (AUD) have prefrontal abnormalities at both 
structural (De Bellis et al., 2005; Medina et al., 2008) and functional levels (Caldwell et al., 2005; Tapert, Pulido, Paulus, Schuckit, \& Burke, 2004). Likewise, population-based studies have shown that adolescent BDs have thicker cortices in left frontal regions (Squeglia et al., 2012a), possibly indicating reduced synaptic pruning and less neurodevelopment. In a study carried out by our research group with part of this sample, BDs were found to have a larger volume of grey matter in the left middorsolateral prefrontal cortex (mid-DLPFC) than nonBDs (Doallo et al., 2014). Such brain alterations have been interpreted in terms of a neuromaturational delay related to heavy alcohol consumption during adolescence. At the functional level, increased frontal activity during performance of WM tasks has been observed in BDs (Campanella et al., 2013; Squeglia, Schweinsburg, Pulido, \& Tapert, 2011; Squeglia et al., 2012b), suggesting compensatory mechanisms and greater effort to perform the task at the same level as the non-BD counterparts.

At the neuropsychological level, WM has been one of the cognitive processes most commonly investigated in relation to young BDs (López-Caneda et al., 2014a). Three studies by the same research group (García-Moreno, Torrejón, Sanhueza, \& Carrére, 2008; GarcíaMoreno, Expósito, Sanhueza, \& Gil, 2009; Sanhuenza, García-Moreno, \& Expósito, 2011) found that BDs performed poorly in WM tasks (Digit Span [WMS-III] and Corsi block tapping task [CBTT]). In relation to the executive processes of WM, female BDs committed more errors in the most difficult condition of the Spatial Working Memory task (Cambridge Neuropsychological Test Automated Battery [CANTAB]) and obtained lower strategy scores (Scaife \& Duka, 2009; Townshend \& Duka, 2005). However, other authors have not found any differences regarding number of errors in a similar test, the Self Ordered Pointing Task (SOPT) (Johnson et al., 2008; Xiao et al., 2009). Previous studies by our research group have shown that BDs had lower span in the Backward Digit test (Digit Span [WMS-III]) (Parada et 
al., 2012) and committed more perseverative errors in the SOPT (Mota et al., 2013; Parada et al., 2012) than non-BDs. The results with part of this sample showed that the differences in Digits Backward disappeared despite maintenance of a BD pattern of alcohol consumption during two years (Mota et al., 2013). This apparent improvement in verbal WM in BDs in comparison with age-matched non-BDs may be compatible with a neuromaturational lag, possibly indicating that improvements in WM efficiency that take place naturally during adolescence would occur later in BDs as a result of excessive alcohol consumption.

Despite the consistent deficits in WM, little is known about the long-term progression of such difficulties in relation to BD trajectories. A recent 8-year-long prospective study (Boelema et al., 2015) did not find any association between executive functions and BD trajectory in young adults. However, the authors used simple reaction time scores as indexes of cognitive processes (e.g. WM), which may lead to poor identification of subtle difficulties (e.g. manipulation, perseveration). In a slightly different population, a ten-year-long followup study of adolescents with alcohol and other drug use (AOD) showed that different patterns of substance use were linked to poor verbal learning and memory, visuospatial memory and WM over time (Hanson, Cummins, Tapert, \& Brown, 2011), and heavier use was generally related to poor performance. In addition, there is a further gap in the literature in relation to whether recovery would occur after abandonment of the BD pattern of alcohol consumption in the short-term, and if so, whether the ex-BDs would be able to perform at the same level as controls.

The main aim of the present study was to determine the relationship between WM and BD trajectory over a 6-year period in healthy university students with no other relevant risk factors, such as psychiatric comorbidity or family history of alcoholism. Bearing in mind that this pattern is associated with difficulties in specific WM processes (maintenance of 
information, manipulation, resistance to interference, etc.), we placed particular emphasis on disentangling some these processes by considering different variables not previously analysed. We also explored what happens when memory load increases (more items to monitor) and executive strategies are required to complement storage limits. Moreover, as our previous results showed amelioration of difficulties in the digit span task after two years of a stable BD pattern of alcohol consumption (Mota et al., 2013), we hypothesized that improvements in WM would be observed and would suggest alcohol-related delays in normal adolescent neuromaturational processes.

We tested the following hypotheses: (I) participants with a trajectory of stable BD will show poorer performance in high-demanding blocks (when they might not be able to compensate their difficulties) of WM task than stable non-BDs, which may be consistent with a compensatory hypothesis; (II) despite maintenance of the pattern, stable-BDs will show an improvement in WM over time, compatible with a neuromaturational delay in brain development; and (III) performance of this task by those participants who abandon the BD pattern of alcohol consumption will improve, and maintenance of an ex-BD pattern over time would imply further improvement.

\section{Material and methods}

\section{Participants}

The sample initially comprised 155 first-year university students (76 males and 79 females) recruited from different faculties of the University of Santiago de Compostela (NW Spain). All participants were Caucasian. Participants were selected by administration of an anonymous questionnaire in class (Caamaño-Isorna, Corral, Parada, \& Cadaveira, 2008). The questionnaire included the Alcohol Use Disorders Identification Test (AUDIT) (Babor, 
Higgins-Biddle, Saunders, Monteiro, 2001) and questions related to alcohol use (speed of consumption, age of onset, etc.). The classification criteria were based on the students' responses to two questions: the third item of the AUDIT (How often do you have six or more drinks on a single occasion? Never/Less than monthly/Monthly/Weekly/Daily or almost daily) and one question related to the speed of consumption (drinks per hour). Taking into account that in Spain a standard alcoholic drink is equivalent to $10 \mathrm{~g}$ of alcohol, six drinks consumed at a speed of more than two drinks per hour would bring the blood alcohol concentration (BAC) to $0.8 \mathrm{~g} / \mathrm{l}$ or higher. This criterion defined a binge drinking pattern of alcohol consumption in the present study. Thus, BD participants consumed 6 drinks on one occasion monthly or weekly and the speed of alcohol consumption was three drinks or more per hour. The non-BD group was defined as those who never consumed 6 drinks on one occasion (or less than monthly) and they consumed alcohol at a speed of two drinks or less per hour.

As the objective was to assess the trajectory of alcohol consumption, participants were classified as stable non-BDs, stable BDs and Ex-BDs (those who abandoned the BD pattern at the second, third or fourth evaluation). This classification criterion was strict and did not allow transitions or fluctuations in the trajectories (e.g. a non-BD participant who changed to a BD consumption at the third evaluation would be excluded from the analysis in the fourth evaluation but maintained for the previous evaluations thanks to the ability of mixed models to deal with different number of participants in each evaluation). We are aware that this classification implies sample attrition over time. Therefore, to ensure that this method did not influence the results, we performed a statistical analysis allowing transitions in consumption trajectory (e.g. a non-BD in the first evaluation who changed to a BD in the second assessment was then considered within this new group at that specific time point). In 
other words, the statistical model considered the specific pattern of consumption at each time point. Use of this type of classification implies less sample attrition over time; however, the results obtained were almost identical. Thus, for the sake of simplicity, we used the stable group classification. Abstainers were not included in the study.

The number of participants decreased throughout the study: 155 (76 non-BDs/ 79 BDs) participants at baseline, 93 (39 stable non-BDs/ 33 stable BDs/ 21 ex-BDs) in the first follow-up, 74 (33 stable non-BDs/ 17 stable BDs/ 24 ex-BDs) in the second and 40 (16 stable non-BDs/ 4 stable BDs/ 20 ex-BDs) in the final follow-up. The number of total data points in each trajectory was 164 for stable non-BDs, 133 for stable-BDs and 65 for ex-BDs. There were no statistically significant differences between those participants included in the final follow-up and those who abandoned the study or were excluded at different times throughout the study, in relation to any of the parameters considered (age of onset of alcohol use, pattern of alcohol consumption and psychopatological symptoms).

\section{Procedure}

Once classified according to alcohol consumption patterns, participants were interviewed to obtain information about their clinical and sociodemographic status. To reduce the potentially confounding effects of other factors, the following exclusion criterion was used: consumption of any other drugs (e.g. opiates, hallucinogens, cocaine, amphetamines or medically prescribed psychoactive substances), except nicotine and cannabis. The item in the questionnaire regarding drug use was "Which of these substances have you consumed and how frequently do you consume them?". Other exclusionary criteria included the following: diagnosis of alcohol use disorders; severe non-corrected motor or sensory deficits; family history of major mental disorder; history of alcoholism in first- and second-degree relatives; history of psychopathology (DSM-IV-TR), such as attention-deficit hyperactivity disorder, 
conduct disorder or previous diagnosis of depression or anxiety; and current psychopathological symptoms as assessed by the Symptom Checklist-90-R (SCL-90-R) (Derogatis, 1983). Participants were excluded if they had scores above 90th in the Global Severity Index (GSI) or in at least two symptomatic dimensions.

Evaluations were made on average every 22 months, and a total of four evaluations were carried out. At each new evaluation we only contacted participants who were included in the previous evaluation: participants in the final evaluation were thus included in all previous assessments. In addition, during each evaluation a neuropsychological battery of tests was administered together with an interview in which the same exclusionary criteria were considered again in order to produce a sample of healthy university students with no other risk factors, avoiding possible confounding factors. All participants gave written informed consent and received some monetary compensation (i.e. $30 €$ at each follow-up). The research was performed in accordance with the ethical principles for research involving human subjects.

\section{Material}

Self-Ordered Pointing Test, abstract design version (SOPT) (Petrides \& Milner, 1982). This test consists of a booklet of abstract designs repeated on all pages but with a different position on each new page. The participant is asked to point out a different stimulus on each page without repeating previous choices. The test is divided into four blocks of increasing difficulty $(6,8,10$ and 12 stimuli to monitor), and each block consists of 3 trials (108 sheets in total). The following variables were recorded for each participant: total number of errors, perseverative errors (pointing to the same item chosen on the immediately preceding page), span in each block and trial (number of new elements identified before the first error), monitoring (total number of errors that occurred from the third page onwards 
throughout blocks three and four), proactive interference ([(total errors in the $3^{\circ}$ trial in blocks 3 and 4 ) - (total errors in $1^{\circ}$ trial blocks 3 and 4$\left.)\right]$ / [(total errors in the $3^{\circ}$ trial blocks 3 and 4) $+\left(\right.$ total errors in the $1^{\circ}$ trial blocks 3 and 4)]). The score was categorized into four values ranging from -1 to 1 , to deal with negative values. The SOPT assesses planning and self-monitoring aspects of WM. Research including studies on non-human primate lesions (Petrides, 1991, 1995) and human imaging studies (Curtis et al., 2000; Petrides, 2000) highlight the importance of DLPFC (Brodmann's areas [BA] 46 and 9) to successful performance of this task.

\section{Statistical analysis}

Generalized linear mixed models (GLMMs), in which maximum log-likelihood was approximated by adaptive Gauss-Hermite quadrature, were used in the statistical analysis (Brown \& Prescott, 2006). Use of GLMMs allows analysis of repeated measurements (measurement correlation) with different number of participants in each evaluation, yields greater statistical power than ordinary regression models and can better handle the problems inherent to longitudinal data (Gibbons, Hedeker, \& DuToit, 2010). To construct the models, we used the scores of the SOPT as dependent variables, with individual observations as level 1 and students as level 2; random effects among students were considered to control the initial intra-individual heterogeneity. In view of the fact that the response variables were test scores, these models were fitted with the Poisson family of distributions, although perseverative errors were fitted with a negative binomial distribution to obtain standard errors corrected for the overdispersion parameter. The models were adjusted for time and sex. The interaction of these variables with the trajectory of alcohol consumption was also modelled. Frequency of cannabis use, frequency of tobacco use, age of drinking onset and GSI score of the SCL-90-R were tested to determine whether they had an explanatory role. The 
independent variables with a statistical significance lower than 0.2 at a bivariate level were included in the multivariate models. The nonsignificant independent variables were eliminated from this maximum model when the coefficients of the main exposure variables did not vary by more than $10 \%$ and the value of Schwartz's Bayesian Information Criterion (BIC) decreased.

All analyses were performed using the free R (version 3.1.1) statistical software environment (R Core Team, 2015) with the lme4 package (Bates, Maechler, Bolker, \& Walker, 2014), and all results were expressed as relative risks (RRs) and their $95 \%$ confidence intervals (CIs). In order to observe the progression of deficits over time, we constructed GLMMs separately for the stable BDs and non-BDs. This model enabled us to identify the progression in WM perforrmance for both groups at each time point relative to baseline.

\section{Results}

\section{Demographic and substance use variables}

The descriptive characteristics of the sample at baseline are shown in Table 1. Groups differed in age, $t(152)=2.86, p=.005$. The BDs were slightly older than the non-BDs. The groups did not differ in estimated intellectual level assessed by the Vocabulary subtest (WAIS-III) (Wechsler, 1997). There were no differences between groups in psychopathological symptoms measured by GSI scores of the SCL-90-R test, $t(153)=0.76$, $p=.447$. The groups differed in the following variables: age of onset of alcohol use, $t(137)=$ 4.83, $p=.001$; total AUDIT scores, $t(124.32)=15.68, p=.001$; number of drinks per hour, $t(153)=14.48, p=.001 ;$ alcohol grams consumed during the week, $t(73.61)=8.44, p=.001$; and alcohol grams consumed on the day of highest consumption, $t(71.51)=5.94, p=.001$. 
Between-group differences were also found in cannabis use, $\mathrm{X}^{2}(2, \mathrm{~N}=153)=19.50, p=.001$, and tobacco use, $\mathrm{X}^{2}(2, \mathrm{~N}=153)=8.12, p=.004$. None of the participants included in the study consumed cannabis daily. Eight participants were considered occasional users, as five of them consumed cannabis monthly and three of them consumed cannabis weekly. The other participants consumed (if at all) cannabis less frequently than once a month.

\section{INSERT TABLE 1}

\section{Binge drinking trajectory}

The statistical models for trajectories of consumption considered the total number of participants in each trajectory (throughout all evaluations) (Table 2). The analysis revealed a significant association between stable BD trajectory and perseverative errors in the SOPT (Relative risk $[\mathrm{RR}]=1.45,95 \% \mathrm{CI}[1.05,2.00], p=.024)$. The results showed a lower WM span in stable BDs than in stable non-BDs in the first trial of the third block (with 10 items to monitor $)(\mathrm{RR}=0.90,95 \% \mathrm{CI}[0.82,0.99], p=.030)$ and in the third trial of the fourth block (with 12 items) $(\mathrm{RR}=0.92,95 \% \mathrm{CI}[0.84,0.99], p=.043)$ (Table 2). There were no sex-bygroup interaction effects. The statistical models controlled for frequency of cannabis use, frequency of tobacco use, age of alcohol onset and the GSI score of the SCL-90-R. None of the variables was significantly associated with performance on the task (at the bivariate or multivariate level).

Regarding abandonment of the pattern of alcohol consumption, the Ex-BDs participants did not differ from stable non-BDs in any of the variables (see Table 2). There were also no significant differences between the stable BDs and Ex-BDs. The effect of duration of abstinence (participants who recently abandoned the BD pattern relative to those 
who had a long-term maintenance of non-BD consumption, both relative to the stable nonBDs) was not significant.

\section{INSERT TABLE 2}

\section{Changes in performance of stable BDs}

In order to determine any changes in performance, we conducted a separate analysis for the stable BDs, with time as a covariable. The relative risk at each follow-up relative to baseline is shown in Table 3 . The number of perseverative errors did not differ significantly over time. Regarding the WM span, no significant effects were found for the first block or the second block. However, stable BDs showed some improvement in WM span at the first follow-up (fourth block, third trial, $\mathrm{RR}=1.19,95 \% \mathrm{CI}[1.04,1.37], p=.012$ ) and at the second follow-up, in the third block, first trial ( $\mathrm{RR}=1.23,95 \% \mathrm{CI}[1.01,1.49, p=.037])$ and fourth block, first trial $(\mathrm{RR}=1.23,95 \% \mathrm{CI}[1.03,1.46], p=.019)$ relative to baseline. Analysis of the changes in performance in the stable non-BD group revealed significant improvements at the second follow-up relative to baseline, in the fourth block of the second trial $(\mathrm{RR}=1.23$, $95 \%$ CI $[1.05,1.43], p=.041)$.

\section{INSERT TABLE 3}

\section{Discussion}

The main aim of this study was to analyze the association between BD trajectory and WM in healthy university students with no other relevant risk factors throughout the 6-year study period (i.e. between ages of 18-19 years and 24-25 years). We first hypothesized that participants with a stable BD pattern would perform poorly for WM in the SOPT, in comparison with stable non-BDs, particularly in high-demanding conditions when compensatory mechanisms may no longer be successful. The results confirm that a stable BD 
trajectory is associated with WM deficits, specifically more perseverative errors in total (independently of block difficulty) and poor WM span when the difficulty of the task increased. Stable BDs displayed a lower WM span only in high-demanding blocks of the SOPT. Stable BDs appear able to compensate for the difficulties up to a certain point (e.g. blocks with 10 items), when correct manipulation of items requires use of strategies. This may indicate that a stable BD trajectory is related to manipulation deficits when difficulty is increased rather than to a simple maintenance problem.

In the general population, abnormal functional activity has been found in adolescent BDs on WM tasks, even in the absence of any differences in performance, which may indicate compensatory mechanisms and a greater effort to solve the task efficiently (Campanella et al., 2013; Squeglia 2011, 2012b). These findings may be consistent with the lower WM span scores obtained only for the most demanding blocks in this study.

Compensatory mechanisms may thus enable stable BDs to perform correctly in low-demand blocks. In a previous study by our research group, electrophysiological findings have revealed anomalies in neural activity involving WM/attention processes after two years of a stable BD. These results may reflect the recruitment of additional resources for adequate task performance and may compensate underlying dysfunctions (López-Caneda et al., 2013).

At a neuropsychological level, two studies (Scaife \& Duka, 2009; Townshend \& Duka, 2005) found that female BDs made more errors in the most difficult condition of an equivalent task, the Spatial Working Memory task (CANTAB). However, other studies using the SOPT have found that controls and BDs perform similarly (Johnson et al., 2008; Xiao et al., 2009). Interestingly, this may be related to the fact that perseverative errors or WM span variables were not recorded and that these variables seem to be more sensitive to weaknesses in the executive process of the WM. To our knowledge, to date only one study has examined 
the relationship between BD trajectory and WM in university students with no other risk factors and during a long period (Boelema et al., 2015). In the aforementioned study the students were followed throughout eight years and were subjected to four neuropsychological assessments. The authors found no association between executive functions and BD. However, the study may have been limited by the fact that the authors used simple reaction time scores as indices of cognitive processes (e.g. WM), and these probably do not identify subtle difficulties (e.g. manipulation, perseveration). Another prospective study involving a clinical sample of adolescents with alcohol and other drug use (AOD) (Hanson et al., 2011) found that different patterns of substance use were associated with poor WM, among other cognitive functions. The performance of chronic AOD users deteriorated more steadily over time in WM.

In a previous study with part of the same sample as used in the present study, we found differences in Digit Backward span (Parada et al., 2012) at baseline; however, interestingly, these differences had already disappeared at the first follow-up (Mota et al., 2013). In both of these studies, the BDs also made more perseverative errors in the SOPT, but no other variables, apart from total errors, were considered. In addition, a neuroimaging study with part of the same sample (Doallo et al., 2014) found a larger volume of grey matter in the left mid-DLPFC (Brodmann areas 46 and 9) in BDs than in control subjects; this was interpreted as neuromaturational delay. A significant positive correlation between left midDLPFC volume and total errors in the SOPT was also found in BDs. We therefore hypothesized that neuropsychological difficulties will show a certain degree of improvement, compatible with a neuromaturational delay related to the effects of alcohol consumption. In this regard, we observed two slightly different trends in how these difficulties progressed. On the one hand, WM span improve in stable BDs relative to the scores at baseline. This may 
support a neuromaturational lag affecting executive functions, as BDs seem to gain efficiency in WM later than non-BDs. Neuromaturational immaturity related to the deleterious effects of alcohol may also explain why other WM deficits present during the first evaluations disappear/recover even when the BD pattern is maintained. Performance of tasks largely mediated by the DLPFC mature latest (Gogtay et al., 2004) and, of these, the cognitive underpinnings of tasks that demand high levels of executive control mature later than those that require WM but decreased control (e.g. SOPT versus backward span tasks; Luciana, Conklin, Hooper, \& Yarger, 2005). In turn, such impairments may be more visible in middle adolescence (18-19 years, baseline), when neurodevelopment is undergoing significant changes.

On the other hand, stable BDs committed more perseveration errors than non-BDs throughout the study. Furthermore, no difference in performance was observed over time in relation to this deficit. In other words, the risk associated with perseverations remained stable in those BDs who maintained a pattern of excessive alcohol consumption. As we did not analyze the neuropsychological performance prior to the onset of the pattern of alcohol consumption, this could be interpreted either as a consequence of BD or as indicating a preexisting vulnerability. In this regard, two recent studies (Peteers, Monshouwer, Janssen, Wiers, \& Vollebergh, 2014; Peeters et al., 2015) suggested that poor WM functioning in the SOPT (mean of the proportion of errors and mean of correct responses) constitutes both a risk factor for heavy drinking and a function that is negatively influenced by alcohol. However, the conclusions of these studies must be considered with caution as the sample comprised adolescents with externalizing problems. Although chronic alcoholism is known to be related to poor executive functions such as WM deficits and perseveration (Bernardin, MaheutBosser, \& Paille, 2014), few studies have investigated perseveration in young adult BDs. 
Regarding the third hypothesis, we did not find any differences between Ex-BDs and stable non-BD, or between ex-BDs and their stable BD counterparts. Abandoning the BD pattern of consumption, even recently, is associated with average performance in the SOPT. It seems that WM deficits (perseveration and span weakness) are not long-lasting consequences once the $\mathrm{BD}$ pattern of alcohol consumption is abandoned. These findings may indicate partial recovery of the deficits in the short-term. This is consistent with other studies carried out by our research group (López-Caneda, Holguín, Corral, Doallo, \& Cadaveira, 2014b; Mota et al., 2013), in which Ex-BDs displayed an intermediate position between the two groups (non-BDs and BDs) in cognitive performance.

The main limitation of this longitudinal study is the sample attrition. This mainly affected analysis of the progression of stable BDs over time (each follow-up compared with baseline), especially at the final follow-up (in which no statistically significant differences were observed). Nonetheless, GLMMs are particularly useful in longitudinal designs, offering the advantage of handling different number of participants in each evaluation. This means that a participant evaluated three times but not included in the final evaluation was included in the analysis up to that point. Thus, the findings related to the stable trajectories of consumption are less affected by this limitation, as a greater number of data points are considered in each trajectory. Furthermore, these models also deal with the response correlation in repeated measures (such as correlated measurement errors and participant's heterogeneity), resulting in greater statistical power (Gibbons et al., 2010).

Another possible limitation of the present study is the fact that we did not carry out a neuropsychological assessment of the participants before the onset of drinking. Thus, we cannot determine the extent to which some of the cognitive deficits were already present before the onset of alcohol use. Several functional studies have suggested the existence of 
some different brain activity prior to the onset of substance use, constituting a risk factor, and that once initiated, heavy drinking may lead to additional brain alterations (Norman et al., 2011; Squeglia et al., 2012b; Wetherill, Squeglia, Yang, \& Tapert, 2013). Importantly, deficits in WM may lead to a vicious circle of BD and further deficits, with an associated greater likelihood of maintenance or escalation of heavy drinking (Peeters et al., 2014, 2015). In this sense, possible intervention strategies for stable BDs who persist with this pattern into adulthood may benefit from WM training (as suggested by Houben, Wiers, \& Jansen, 2011).

These findings are of particular importance not only because WM is a central aspect of reasoning, planning and decision-making (Diamond, 2013), but also because deficits in WM may lead to alcohol-related problems in the future (Peeters et al., 2014). In conclusion, the results of the present longitudinal study revealed that maintenance of a BD pattern of alcohol consumption, even in university students with no other risk factors, is associated with neuropsychological deficits in WM, particularly perseveration deficits and low WM span over time. Regarding the progression of deficits, perseveration difficulties remained stable, while certain improvements in WM were visible despite maintenance of the BD pattern. Abandoning the pattern of alcohol consumption led to similar performance as in non-BDs, which may be a sign of partial recovery.

\section{Acknowledgements}

The study was supported by grants from the Spanish Ministerio de Ciencia e Innovación (PSI2011-22575). Carina Carbia was supported by the FPU program (FPU13/04569) of the Spanish Ministerio de Educación.

\section{References}


Babor, T. F., Higgins-Biddle, J. C., Saunders, J. B., Monteiro, M. G. (2001). AUDIT. The Alcohol Use Disorders Identification Test. Guidelines for Use in Primary Health Care, 2nd ed. World Health Organization, Geneva.

Bates, D., Maechler, M., Bolker, B., \& Walker, S. (2014). _lme4: Linear mixed-effects models using Eigen and S4_. $\mathrm{R}$ package version 1.1-7, <URL: http://CRAN.Rproject.org/package=lme4>.

Bava, S., \& Tapert, S. F. (2010). Adolescent brain development and the risk for alcohol and other drug problems. Neuropsychology Review, 20(4), 398-413. doi: 10.1007/s11065-010-9146-6

Bernardin, F., Maheut-Bosser, A., \& Paille, F. (2014). Cognitive impairments in alcoholdependent subjects. Frontiers in Psychiatry, 5, 78. doi: 10.3389/fpsyt.2014.00078

Boelema, S. R., Harakeh, Z., van Zandvoort, M. J., Reijneveld, S. A., Verhulst, F. C., Ormel, J., \& Vollebergh, W. A. (2015). Adolescent heavy drinking does not affect maturation of basic executive functioning: longitudinal findings from the TRAILS study. PloS ONE, 10(10). doi: 10.1371/journal.pone.0139186

Brown, H., \& Prescott, R. (2006). Applied Mixed Models in Medicine. New York: John Wiley \& Sons.

Caamaño-Isorna, F., Corral, M., Parada, M., \& Cadaveira, F. (2008). Factors associated with risky consumption and heavy episodic drinking among Spanish university students. Journal of Studies on Alcohol and Drugs, 69(2), 308-312. doi: 10.15288/jsad.2008.69.308

Caldwell, L. C., Schweinsburg, A. D., Nagel, B. J., Barlett, V. C., Brown, S. a., \& Tapert, S. F. (2005). Gender and adolescent alcohol use disorders on BOLD (blood oxygen level 
dependent) response to spatial working memory. Alcohol and Alcoholism, 40(3), 194-200. doi: 10.1093/alcalc/agh134

Campanella, S., Peigneux, P., Petit, G., Lallemand, F., Saeremans, M., Noël, X., ... Verbanck, P. (2013). Increased cortical activity in binge drinkers during working memory task: a preliminary assessment through a functional magnetic resonance imaging study. PLoS ONE, 8(4). doi: 10.1371/journal.pone.0062260

Crews, F. T., Braun, C. J., Hoplight, B., Switzer, R. C., \& Knapp, D. J. (2000). Binge ethanol consumption causes differential brain damage in young adolescent rats compared with adult rats. Alcoholism: Clinical and Experimental Research,24(11), 1712-1723. doi: 10.1111/j.1530-0277.2000.tb01973.x

Curtis, C. E., Zald, D. H., \& Pardo, J. V. (2000). Organization of working memory within the human prefrontal cortex: a PET study of self-ordered object working memory. Neuropsychologia, 38(11), 1503-1510. doi:10.1016/S0028-3932(00)00062-2

De Bellis, M. D., Narasimhan, A., Thatcher, D. L., Keshavan, M. S., Soloff, P., \& Clark, D. B. (2005). Prefrontal cortex, thalamus, and cerebellar volumes in adolescents and young adults with adolescent-onset alcohol use disorders and comorbid mental disorders. Alcoholism, Clinical and Experimental Research, 29(9), 1590-1600. doi: 10.1097/01.alc.0000179368.87886.76

Derogatis, L. R. (1983). SCL-90-R. Administration, scoring and procedures manual II for the revised version of the SCL-90-R. Baltimore: John Hopkins University Press.

Diamond, A. (2013). Executive Functions. Annual Review of Psychology, 64, 135-168. doi: 10.1146/annurev-psych-113011-143750 
Doallo, S., Cadaveira, F., Corral, M., Mota, N., López-Caneda, E., \& Rodríguez Holguín, S. (2014). Larger mid-dorsolateral prefrontal gray matter volume in young binge drinkers revealed by voxel-based morphometry. PLOS ONE, 9(5). doi: 10.1371/journal.pone.0096380

Eurobarometer (2010). EU citizens' attitudes towards alcohol. Special Eurobarometer 331 / Wave 72.3. Brussels: TNS Opinion \& Social at the request of Directorate General Health and Consumers and coordinated by the Directorate-General for Communication of European Commission.

Fuster, J. M. (2001). The prefrontal cortex — an update: time is of the essence. Neuron, 30(2), 319333. doi:10.1016/S0896-6273(01)00285-9

García-Moreno, L. M., Expósito, J., Sanhueza, C., \& Gil, S. (2009). Rendimiento cognitivo y consumo de alcohol durante los fines de semana en mujeres adolescentes. Revista Neuropsicología, Neuropsiquiatría y Neurociencias, 9, 75-91

García-Moreno, L. M. G., Torrejón, F. J. E., Sanhueza, C., \& Carrére, M. T. A. (2008). Actividad prefrontal y alcoholismo de fin de semana en jóvenes. Adicciones: Revista de socidrogalcohol, 20(3), 271-279.

Gibbons, R. D., Hedeker, D., \& DuToit, S. (2010). Advances in analysis of longitudinal data. Annual review of Clinical Psychology, 6, 79-107. doi: 10.1146/annurev.clinpsy.032408.153550

Gogtay, N., Giedd, J. N., Lusk, L., Hayashi, K. M., Greenstein, D., Vaituzis, A. C., ... Thompson, P. M. (2004). Dynamic mapping of human cortical development during childhood through early adulthood. Proceedings of the National Academy of Sciences of the United States of America, 101(21), 8174-8179. http://doi.org/10.1073/pnas.0402680101 
Hanson, K. L., Cummins, K., Tapert, S. F., \& Brown, S. A. (2011). Changes in neuropsychological functioning over 10 years following adolescent substance abuse treatment. Psychology of Addictive Behaviors, 25(1), 127-142. doi: 10.1037/a0022350

Houben, K., Wiers, R. W., \& Jansen, A. (2011). Getting a grip on drinking behavior training working memory to reduce alcohol abuse. Psychological Science. 22(7), 968-75. doi: $10.1177 / 0956797611412392$

Jacobus, J., \& Tapert, S. F. (2013). Neurotoxic effects of alcohol in adolescence. Annual Review of Clinical Psychology, 9, 703-721. doi:10.1146/annurev-clinpsy-050212-185610.

Johnson, C. A., Xiao, L., Palmer, P., Sun, P., Wang, Q., Wei, Y., ... Bechara, A. (2008). Affective decision-making deficits, linked to a dysfunctional ventromedial prefrontal cortex, revealed in 10th grade Chinese adolescent binge drinkers. Neuropsychologia, 46, 714-726. doi:10.1016/j.neuropsychologia.2007.09.012

López-Caneda, E., Cadaveira, F., Crego, A., Doallo, S., Corral, M., Gómez-Suárez, A., \& Holguín, S. R. (2013). Effects of a persistent binge drinking pattern of alcohol consumption in young people: a follow-up study using event-related potentials. Alcohol and alcoholism, 48(4), 464-471. doi: 10.1093/alcalc/agt046

López-Caneda, E., Mota, N., Crego, A., Velasquez, T., Corral, M., Holguín, S. R., \& Cadaveira, F. (2014a). Anomalías neurocognitivas asociadas al consumo intensivo de alcohol (binge drinking) en jóvenes y adolescentes: Una revisión. Adicciones, 26(4), 334-359. doi: http://dx.doi.org/10.20882/adicciones.39

López-Caneda, E., Holguín, S. R., Corral, M., Doallo, S., \& Cadaveira, F. (2014b). Evolution of the binge drinking pattern in college students: Neurophysiological correlates. Alcohol, 48(5), 407-418. doi: 10.1016/j.alcohol.2014.01.009 
Luciana, M., Conklin, H. M., Hooper, C. J., \& Yarger, R. S. (2005). The development of nonverbal working memory and executive control processes in adolescents. Child development, 76(3), 697-712. doi: 10.1111/j.1467-8624.2005.00872.x

Marshall, E. J. (2014). Adolescent alcohol use: Risks and consequences. Alcohol and Alcoholism, 49(2), 160-164. doi: 0.1093/alcalc/agt180

Medina, K. L., McQueeny, T., Nagel, B. J., Hanson, K. L., Schweinsburg, A. D., \& Tapert, S. F. (2008). Prefrontal cortex volumes in adolescents with alcohol use disorders: unique gender effects. Alcoholism, Clinical and Experimental Research, 32(3), 386-394. doi: 10.1111/j.1530-0277.2007.00602.x

Mota, N., Parada, M., Crego, A., Doallo, S., Caamaño-Isorna, F., Rodríguez Holguín, S., ... Corral, M. (2013). Binge drinking trajectory and neuropsychological functioning among university students: A longitudinal study. Drug and Alcohol Dependence, 133(1), 108-114. doi: 10.1016/j.drugalcdep.2013.05.024

National Institute of Alcohol Abuse and Alcoholism (2004). NIAAA council approves definition of binge drinking. NIAAA Newsletter, $3, \quad$ p. 3. http://pubs.niaaa.nih.gov/publications/Newsletter/winter2004/Newsletter_Number3.pdf

Norman, A. L., Pulido, C., Squeglia, L. M., Spadoni, A. D., Paulus, M. P., \& Tapert, S. F. (2011). Neural activation during inhibition predicts initiation of substance use in adolescence. Drug and alcohol dependence, 119(3), 216-223. doi: 10.1016/j.drugalcdep.2011.06.019

Parada, M., Corral, M., Mota, N., Crego, A., Rodríguez Holguín, S., \& Cadaveira, F. (2012). Executive functioning and alcohol binge drinking in university students. Addictive Behaviors, 37(2), 167-172. doi:10.1016/j.addbeh.2011.09.015 
Pascual, M., Pla, A., Miñarro, J., \& Guerri, C. (2014). Neuroimmune activation and myelin changes in adolescent rats exposed to high-dose alcohol and associated cognitive dysfunction: a review with reference to human adolescent drinking. Alcohol and alcoholism, 49(2), 187-192. doi: 10.1093/alcalc/agt164

Peeters, M., Janssen, T., Monshouwer, K., Boendermaker, W., Pronk, T., Wiers, R., \& Vollebergh, W. (2015). Weaknesses in executive functioning predict the initiating of adolescents' alcohol use. Developmental cognitive neuroscience. 16, 139-46. doi: 10.1016/j.dcn.2015.04.003

Peeters, M., Monshouwer, K., Janssen, T., Wiers, R. W., \& Vollebergh, W. A. (2014). Working memory and alcohol use in at-risk adolescents: a 2-year follow-up. Alcoholism: Clinical and Experimental Research, 38(4), 1176-1183. doi: 10.1111/acer.12339

Petit, G., Maurage, P., Kornreich, C., Verbanck, P., \& Campanella, S. (2014). Binge drinking in adolescents: A review of neurophysiological and neuroimaging research. Alcohol and Alcoholism, 49(2), 198-206. doi: 10.1093/alcalc/agt172

Petrides, M. (1991). Functional specialization within the dorsolateral frontal cortex for serial order memory. Proceedings of the Royal Society of London. Series B: Biological Sciences, 246(1317), 299-306.

Petrides, M. (1995). Impairments on nonspatial self-ordered and externally ordered working memory tasks after lesions of the mid-dorsal part of the lateral frontal cortex in the monkey. The Journal of Neuroscience, 15, 359-375.

Petrides, M. (2000). The role of the mid-dorsolateral prefrontal cortex in working memory. Experimental Brain Research, 133, 44-54. 
Petrides, M., \& Milner, B. (1982). Deficits on subject-ordered tasks alter frontal and temporal lobe lesions in man. Neuropsychologia, 20, 249-262.

R Core Team (2015). R: A language and environment for statistical computing. R Foundation for Statistical Computing, Vienna, Austria. URL http://www.R-project.org/.

Rehm, J., Mathers, C., Popova, S., Thavorncharoensap, M., Teerawattananon, Y., \& Patra, J. (2009). Global burden of disease and injury and economic cost attributable to alcohol use and alcohol-use disorders. The Lancet, 373(9682), 2223-2233. doi:10.1016/S01406736(09)60746-7

Rubia, K. (2013). Functional brain imaging across development. European Child \& Adolescent Psychiatry, 719-731. doi: 0.1007/s00787-012-0291-8

Sanhueza, C., García-Moreno, L. M., \& Expósito, J. (2011). Weekend alcoholism in youth and neurocognitive aging. Psicothema, 23(2), 209-214.

Scaife, J. C., \& Duka, T. (2009). Behavioural measures of frontal lobe function in a population of young social drinkers with binge drinking pattern. Pharmacology Biochemistry and Behavior, 93(3), 354-362. doi: 10.1016/j.pbb.2009.05.015

Squeglia, L. M., Schweinsburg, A. D., Pulido, C., \& Tapert, S. F. (2011). Adolescent binge drinking linked to abnormal spatial working memory brain activation: differential gender effects. Alcoholism, Clinical and Experimental Research, 35(10), 1831-1841. doi: 10.1111/j.1530-0277.2011.01527.x

Squeglia, L. M., Sorg, S. F., Schweinsburg, A. D., Wetherill, R. R., Pulido, C., \& Tapert, S. F. (2012a). Binge drinking differentially affects adolescent male and female brain morphometry. Psychopharmacology, 220(3), 529-539. doi: 10.1007/s00213-011-2500-4 
Squeglia, L. M., Pulido, C., Wetherill, R. R., Jacobus, J., Brown, G. G., \& Tapert, S. F. (2012b). Brain response to working memory over three years of adolescence: influence of initiating heavy drinking. Journal of Studies on Alcohol and Drugs, 73(5), 749-760

Squeglia, L. M., Spadoni, A. D., Infante, M. A., Myers, M. G., \& Tapert, S. F. (2009). Initiating moderate to heavy alcohol use predicts changes in neuropsychological functioning for adolescent girls and boys. Psychology of Addictive Behaviors, 23(4), 715-722. doi: org/10.1037/a0016516

Substance Abuse and Mental Health Services Administration (2013). Results from the 2012 National Survey on Drug Use and Health: Summary of National Findings, NSDUH Series H46, HHS Publication No. (SMA) 13-4795. Rockville, MD: Substance Abuse and Mental Health Services Administration.

Tapert, S. F., Pulido, C., Paulus, M. P., Schuckit, M. A., \& Burke, C. (2004). Level of response to alcohol and brain response during visual working memory. Journal of studies on alcohol, 65(6), 692-700. doi: 10.15288/jsa.2004.65.692

Townshend, J. M., \& Duka, T. (2005). Binge drinking, cognitive performance and mood in a population of young social drinkers. Alcoholism, Clinical and Experimental Research, 29(3), 317-325. doi: 10.1097/01.ALC.0000156453.05028.F5

Vargas, W. M., Bengston, L., Gilpin, N. W., Whitcomb, B. W., \& Richardson, H. N. (2014). Alcohol Binge Drinking during Adolescence or Dependence during Adulthood Reduces Prefrontal Myelin in Male Rats. Journal of Neuroscience, 34(44), 14777-14782. doi: 10.1523/JNEUROSCI.3189-13.2014

Wechsler D. (1997) Wechsler Adult Intelligence Scale-III. The Psychological Corporation, San Antonio. 
Wetherill, R. R., Squeglia, L. M., Yang, T. T., and Tapert, S. F. (2013). A longitudinal examination of adolescent response inhibition: neural differences before and after the initiation of heavy drinking. Psychopharmacology, 230, 663-671. doi:10.1007/s00213-013$3198-2$

Xiao, L., Bechara, A., Grenard, L. J., Stacy, W. A., Palmer, P., Wei, Y., ... Johnson, C. A. (2009). Affective decision-making predictive of Chinese adolescent drinking behaviors. Journal of the International Neuropsychological Society, 15, 547-557.

doi:10.1017/S1355617709090808 
Table 1

Group means (standard deviation) for demographic and clinical data at baseline.

\begin{tabular}{lcc}
\hline & $\begin{array}{c}\text { Non-BD } \\
(n=76)\end{array}$ & $\begin{array}{c}\text { BD } \\
(n=79)\end{array}$ \\
\hline Sex (m/f) & $36 / 40$ & $40 / 39$ \\
Age** & $18.58(0.60)$ & $18.87(0.63)$ \\
WAIS-III Vocabulary & $12.56(1.97)$ & $12.11(1.62)$ \\
GSI (SCL-90-R), Pc & $46.39(28.83)$ & $50.09(31.36)$ \\
Age of onset alcohol use*** & $15.78(1.04)$ & $14.8(1.30)$ \\
AUDIT total ${ }^{* * *}$ & $2.95(2.58)$ & $12.22(4.55)$ \\
Number of drinks per hour*** & $1.04(0.84)$ & $3.39(1.14)$ \\
Alcohol grams during the week & $42.19(52.79)$ & $302.46(251.13)$ \\
Alcohol grams the day of highest & $27.63(31.93)$ & $166.69(192.12)$ \\
consumption & & 8 \\
Occasional cannabis users*** & 0 & 24 \\
Occasional smokers** & 3 &
\end{tabular}

Note. Five participants consumed cannabis monthly and three of them consumed cannabis weekly.

${ }^{\mathrm{a}}$ The week prior to the evaluation.

${ }^{*} p<.05, * * p<.01, * * * p<.001$ 
Table 2

Relationship between the effect of BD trajectory and scores in the SOPT. GLMMs.

\begin{tabular}{|c|c|c|}
\hline \multicolumn{3}{|c|}{ GLMMs. Relative Risk (95\% CI) } \\
\hline & $\begin{array}{l}\text { Stable BD a } \\
\quad(n=133)\end{array}$ & $\begin{array}{c}\text { Ex-BD a } \\
(n=65)\end{array}$ \\
\hline Total errors & $1.15[0.90,1.47]$ & $1.18[0.90,1.55]$ \\
\hline Monitorization & $1.17[0.92,1.49]$ & $1.25[0.95,1.64]$ \\
\hline Perseverative errors & $1.45[1.05,2.00]^{*}$ & $1.05[0.71,1.57]$ \\
\hline Proactive Interference & $1.00[0.80,1.24]$ & $1.00[0.77,1.29]$ \\
\hline Span $1^{\circ}$ block $1^{\circ}$ trial (6 items) & $0.98[0.88,1.09]$ & $0.97[0.85,1.11]$ \\
\hline $2^{\circ}$ trial & $1.13[0.63,2.03]$ & $0.82[0.40,1.69]$ \\
\hline $3^{\circ}$ trial & $1.00[0.89,1.12]$ & $0.99[0.87,1.14]$ \\
\hline Span $2^{\circ}$ block $1^{\circ}$ trial $(8$ items $)$ & $1.00[0.90,1.11]$ & $1.00[0.88,1.13]$ \\
\hline $2^{\circ}$ trial & $1.00[0.61,1.63]$ & $1.00[0.54,1.86]$ \\
\hline $3^{\circ}$ trial & $1.00[0.90,1.11]$ & $1.00[0.88,1.13]$ \\
\hline Span $3^{\circ}$ block $1^{\circ}$ trial ( 10 items $)$ & $0.90[0.82,0.99]^{*}$ & $0.93[0.83,1.05]$ \\
\hline $2^{\circ}$ trial & $0.94[0.63,1.40]$ & $1.13[0.70,1.82]$ \\
\hline $3^{\circ}$ trial & $0.88[0.74,1.05]$ & $0.88[0.72,1.07]$ \\
\hline Span $4^{\circ}$ block $1^{\circ}$ trial ( 12 items $)$ & $0.93[0.84,1.02]$ & $0.91[0.81,1.02]$ \\
\hline $2^{\circ}$ trial & $1.00[0.65,1.53]$ & $1.00[0.60,1.67]$ \\
\hline $3^{\circ}$ trial & $0.92[0.84,0.99]^{*}$ & $0.97[0.88,1.08]$ \\
\hline
\end{tabular}

Note. This analysis examined working memory performance across 6 years based on binge drinking patterns. CI Confidence interval; GLMM= Generalized linear mixed model; $\mathrm{BD}=$ Binge Drinking; SOPT = Self-Ordered Pointing Test.

${ }^{a}$ Reference category: Stable non-BD trajectory $(n=164)$.

${ }^{*} p<.05, * * p<.01, * * * p<.001$ 
Table 3

Progression of performance in stable BDs in comparison with baseline. GLMMs.

\begin{tabular}{|c|c|c|c|}
\hline & \multicolumn{3}{|c|}{ GLMMs. Relative Risk (95\% CI) } \\
\hline & First follow-up ${ }^{a}$ & Second follow-up ${ }^{a}$ & Third follow-up ${ }^{a}$ \\
\hline Perseverative errors & $0.75[0.54,1.06]$ & $0.88[0.57,1.37]$ & $0.65[0.23,1.82]$ \\
\hline \multicolumn{4}{|c|}{ Span $3^{\circ}$ block ( 10 items) } \\
\hline $1^{\circ}$ trial & $1.10[0.94,1.28]$ & $1.23[1.01,1.49]^{*}$ & $1.28[0.87,1.89]$ \\
\hline $2^{\circ}$ trial & $0.95[0.76,1.19]$ & $0.92[0.63,1.02]$ & $1.14[0.59,2.15]$ \\
\hline $3^{\circ}$ trial & $1.13[0.95,134]$ & $1.03[0.81,129]$ & $1.06[0.68,1.65]$ \\
\hline \multicolumn{4}{|c|}{ Span $4^{\circ}$ block ( 12 items) } \\
\hline $1^{\mathrm{o}}$ trial & $0.99[0.86,1.14]$ & $1.23[1.03,1.46]^{*}$ & $1.03[0.70,1.52]$ \\
\hline $2^{\circ}$ trial & $0.93[0.76,1.14]$ & $0.78[0.56,1.04]$ & $0.74[0.40,1.06]$ \\
\hline $3^{\circ}$ trial & $1.19[1.04,1.37]^{*}$ & $1.14[0.95,1.36]$ & $1.11[0.75,1.64]$ \\
\hline
\end{tabular}

Note. $\mathrm{CI}=$ Confidence interval; GLMM= Generalized linear mixed model.

${ }^{a}$ Reference category: stable BD group performance in baseline. No significant effects were found for $2^{\circ}$ block and $1^{\circ}$ block.

$* p<.05, * * p<.01, * * * p<.001$ 


\section{Highlights:}

- Stable BD during college years is associated to poor working memory (WM).

- Improvement in WM span over time among BDs suggests a neuromaturational delay.

-Stable perseveration difficulties among stable BDs might be related to alcohol consumption or represent a premorbid difference.

-Participants who abandon the BD pattern did not show WM deficits. 